\title{
STONE IN THE UPPER URINARY TRACT.*
}

\author{
By H. P. WINSBURY-WHITE, F.R.C.S. \\ Surgeon, St. Paul's Hospital; Urologist, St. John's Hospital, Lewisham, and \\ Surgeon, Princess Elizabeth of York Hospital for Children.
}

\section{Ætiology.}

I will first say a few words with regard to the ætiology of urinary calculus. One thing which is quite certain is that dietetic errors have a very important influence on urinary lithiasis. We are aware of the fact that urinary stone is common in certain localities throughout the world, and it is the poor in those communities who are afflicted. We find that these people live on a regular, monotonous carbohydrate diet, which not only has those characteristics, but lacks completely food of animal origin. Let us review the known facts in this respect.

In former days, in Western Europe and in America, urinary calculus was a common disease, more common than it is to-day, and the diminished incidence has gone hand-in-hand with an improved standard of living amongst poor people, that improvement taking the form of a change from whole wheat flour to white flour, the consumption of more butter, more milk and perhaps some meat. In India, in the upper Valleys of the Ganges-one of the big areas for stone in the world - the natives subsist largely on a porridge consisting of whole meal. In other stone areas of India, maize is the common diet. But in Madras, though maize is eaten freely, stone is uncommon because fish also is largely taken. In Northern areas of China, where whole wheat meal is the staple food, stone is very common, and in the South where rice takes the place of wheat, stone is also prevalent. In Eastern Europe up to recent times the people have been in more or less the same state as Western Europe was fifty years ago with regard to their daily food, and in Eastern Europe urinary calculus is very common; the same is true of Asia. An interesting fact as to diet in Central Europe is that uroliathiasis has greatly increased in Hanover; this is attributed to the poor food supplied to the children of that locality during the War. In England the replacement of whole wheat flour by white flour has during the past fifty years been accompanied by the gradual diminution of vesical calculus. In this country, when the diet of working people was of so much lower quality and variety than to-day, it was noticed that in Devon, Cornwall, Cheshire and Ireland, where people combined with their potatoes and bread a sufficient quantity of milk and butter, stone was uncommon in comparison with adjacent localities. In Iceland, which is a stonebearing area, the pasture land is not good and the people get little milk and butter. But, moreover, because the grazing is poor, the milk and butter, in those circumstances, are poor in vitamin A. The presence of this food factor is important in the prevention of stone. Another very interesting feature of stone which occurs in a locality where people eat mostly a monotonous carbohydrate diet, is that the stones commonly have uric acid nuclei. Thus it is clear that calculi of this chemical composition are not necessarily dependent upon the metabolism of protein food. There are obviously metabolic changes in the body which are far more intricate than the mere taking of substances which supply a purine basis. 
What experimental evidence is there to support the theory I have stated? Since I9I8 a number of research workers have been engaged on this subject, experimenting largely with rats. The animals have been fed on the diets which are prevalent among the poor communities where stone is common, using as controls animals fed in a different way. The most notable of these researches that have come to my knowledge are those published by McCarrison in I927. In his experiments carried out in India he used hundreds of rats and fed many on a mixed diet such as that used by people who did not get stone, and other large numbers on a diet similar to that of those suffering from stone. A large proportion of the animals which had a monotonous carbohydrate diet developed stone, while not one of the hundreds of control rats contracted the disease. These experiments were very convincing. He went even further in his deductions. He concluded that there were positive factors in the food and that there were negative factors which were responsible. By the latter he meant that there were substances lacking from the food which encouraged lithiasis. The positive factors were, some unknown substance in whole meal, and an excess of calcium. Negative factors included vitamin A of animal origin, and phosphates in sufficient quantity to combine with the amount of calcium present.

We know bilharzia plays its part in stone formation, and that in Egypt and other places it is responsible for a large amount of lithiasis which is present there; and it does not require any particular explanation of this when we realise that the ova are located in the bladder wall, and ultimately escape into the vesical cavity, and thus are free to form nuclei of stone.

Another interesting phase of the xtiology of stone which has been receiving prominence is the effect of excessive sunlight. It is an established fact that prolonged and excessive sunlight produces vitamin D in the body and a result of this is that there is an increased absorption of calcium and phosphate from the intestine. The pigment which exists naturally in the skin of the negro is a protection against this change. And, to bear out this theory, we remember that negroes are very seldom subjects of lithiasis.

There is another aspect of this sunlight theory, and that is that in many tuberculosis sanatoria, where children lie in the sun for long periods, most of them having lesions of bones, a certain proportion develop urinary lithiasis, especially renal calculus. On examining the blood of these patients it is found that the calcium content is higher than normal.

In considering the question of chronic inflammation of bone in its relationship to urinary lithiasis, we also recall that during the War a number of men who were lying with suppurating bone injuries, compound fractures of the femur, etc., were reported to have renal calculus. It was thought that the recumbent posture was responsible for this; but many of them who were not bed ridden developed stone too. As to the war cases, it was not suggested in those days that it might have been due to sunlight. But both in the compound bone injuries and the tuberculous cases chronic osteomyelitis upsets the calcium balance of the blood by liberating this substance from the inflamed bone and possibly that is a reason why lithiasis develops.

We cannot dismiss the possibility that hardness of water has some bearing on the occurrence of stone. This in its turn is due largely to an excess of calcium. In 
Staffordshire when the wells supplied hard water, stone was common. Ultimately, wells were sunk to a depth which provided soft water with the result that the incidence of stone in the district fell considerably.

It is difficult to be certain in any given case such as we see in our daily practice in London, to what extent any of the causes I have mentioned are responsible for the lithiasis. We would therefore be wise to seek for some additional explanation. There is no doubt that inflammation in some part of the body has a very important influence. I remember some research work at the Mayo Clinic six or seven years ago when streptococci were cultivated from the urine of a number of urinary stone cases. Cultures of these were made and implanted in the tooth cavities of six dogs; the excavations were then sealed by amalgam. In a short time five of these dogs developed urinary calculus.

In the urinary tract itself, or in the genital tract which is so closely associated with it, we are frequently coming across evidence of the association of inflammation with urinary lithiasis. I am constantly finding in cases of kidney stone, chronic prostatitis in the male and chronic cervicitis in the female. The commonest organism found is the staphylococcus, and this has the peculiar function of being able to split urea, which is another step towards stone formation.

There is a further important predisposing cause of urinary stone, and that is pre-existing dilatation in some part of the urinary tract. Take hydronephrosis which has resulted from a constriction of the junction of the pelvis and ureter, or the condition seen in children in which the whole upper urinary tract is dilated, that is to say, both ureters and both kidneys, or even the whole urinary tract is involved in the dilatation. In both adults and children I have come across a number in which the dilatation obviously precedes the stone formation. One piece of evidence which strongly indicates this sequence of events is, that at times the dilatation is bilateral in the upper urinary tract, and the stones are only unilateral. There is further evidence by noting that the stones formed inside a large cavity have such a considerable amount of freedom of movement that when they are multiple they remain rounded for a long time. Thus one is able to diagnose by $\mathrm{X}$-rays, stones which have formed in a hydronephrosis. A stone which forms not because of dilatation soon becomes confined in the renal pelvis or a calyx and takes the shape of the cavity which harbours it.

One need make only the briefest reference to the importance of a foreign body in the urinary tract, which will inevitably cause stone if allowed to remain there long enough.

Finally; I would mention the cases of constitutional lithiasis, such as cystine and xanthine stone formation. The pathology which leads to stones of these compositions is not yet understood.

\section{Pathology.}

Now a word about pathology. I have reviewed I62 of my own cases of stone in the upper urinary tract, and I will put before you a few of the facts which this analysis has revealed. I find there was a slight preponderance of males, namely 59 per cent. of the whole. The average age was 38 years. $5 \mathrm{I}$ per cent. were on the left side, 34 per cent. on the right side, and the balance were bilateral cases (I5 per cent.). 
A calculus forms in a renal calyx, and moves from there to the pelvis and from the pelvis to another calyx, according to the action of gravity, until finally the stone, which is increasing in size all the time by the deposition of urinary salts, is of such dimensions that it becomes arrested; the common place for its fixation is the renal pelvis, and it soon assumes the shape of that cavity. As to the amount of damage that a stone can do in the kidney one cannot assess that by the size of the stone. I shall show you at the end of the lecture some small stones which resulted in the complete destruction of the renal tissue; and I have had experience of other stones which have been so large as to make a complete cast of the kidney and yet have caused no dilatation at all. As the obstruction progresses, so the renal tissue becomes thin, the calyces become dilated and there is a progressive diminution of the renal function. In most cases one finds evidence of infection in the urine the moment one comes in contact with the case. In others this evidence is delayed. The onset of infection is sometimes an important item in determining the advent of symptoms. Once infection has established itself and pools of residual urine occur in the dilated calyces the prospects of ever getting rid of the infection are indeed remote. Signs of infection are soon found in the peri-renal tissue. On cutting down on an infected kidney one always finds a peri-nephritis of some degree. There may even be a peri-renal abscess from infection originating as a complication of renal calculus.

As to the chemical composition of stones, I want you to realise that most urinary calculi are a mixture of chemical constituents, some having a preponderance of one constituent, others of another. The chief importance of the chemical com $=$ position of urinary calculi lies in the fact that those composed largely of uric acids or ammonium urate throw poor radiographic shadows, and thus may escapes detection on $\mathrm{X}$-ray examination.

\section{Diagnosis.}

Now a few words about the clinical features and diagnosis of renal calculus. These cases cover a wide variety in regard to clinical features. At one extreme there is very intense pain, perhaps the most excruciating that a human being can experience, that is severe renal colic. Such symptoms are usually caused by a small stone which is in the kidney pelvis and is trying to get down the ureter, or maybe it is actually in the ureter. At the other extreme are cases which have no symptoms. We know that stone is often present in these circumstances because every now and then we see a case in which the patient gives a few weeks' history of symptoms and the X-rays show a stone of such a size that we know has taken years to form without attracting any attention whatever. Then, for some special reason, symptoms develop; usually because an increased degree of infection has developed. Between those two extremes we have the more or less classical cases which come with histories of recurrent attacks of colic, chronic pain between attacks, and hæmaturia having some relationship to the pain. Other cases have only a little hæmaturia, in others there is only pain, often without colic. One may say there are as many cases of renal colic in which the symptom is not due to stone as there are those in which it is due to stone. In other cases the patient simply notices that his water has become thick, thus pyuria may be the first means of calling attention to renal calculus.

As to physical examination in most cases such a kidney is tender, but every now and then a patient with renal calculus shows neither renal tenderness nor enlargement. 
With regard to the most important means of diagnosing renal calculus, radiography is unchallenged. One is not justified in telling a patient he or she has not got stone without the corroboration of an X-ray examination. The method is so accurate that such a responsibility should not be taken without this investigation, in spite of what might appear to be a typical case. A plain X-ray sometimes shows a shadow in the renal region which raises a doubt as to whether the opacity is a stone in the kidney, or whether it is a calcified gland lying outside of the kidney, and one has to pursue the question further. Calcified glands are often multiple and one of their characteristics is that a second X-ray will often show a change of position of the shadows. Another important way. is to turn the patient on to his side and take a lateral radiogram and if it is found that the shadow in question is confluent with the body of the adjacent vertebra, this is diagnostic of stone. One sometimes finds that a stone in the kidney gives a shadow in front of a vertebra. This is usually because the part of the kidney which contains the stone is pushed forward as a result of enlargement; and it will thus show in front of the line of the vertebral bodies.

\section{Treatment.}

I must now refer briefly to treatment. There are certain renal stone cases in which operation is contra-indicated. For instance, where there is extensive bilateral renal lithiasis in which the stones are widespread in both kidneys. Nor does one operate on cases in which the X-rays show a small stone of such a size that it has a good chance of passing down the ureter. One watches such a case by means of repeated X-ray examinations, and if the patient has occasional colic, all the better, it shows that the stone is moving down the ureter. One learns from experience on seeing the size of the shadow whether the patient is likely to get rid of the stone spontaneously. A shadow half the size of a split pea, or a little larger is a case which should not be operated upon, the patient should be given every opportunity of getting rid of the calculus per vias naturales. In old or feeble people, independently of bilateral stone, one would not, as a rule, operate unless there is some pressing emergency.

We must never undertake operative interference without first of all having a full knowledge of the condition, not only of the diseased kidney, but also of the opposite one. One carries out cystoscopy and collects the urine from that kidney and examines it to see if pus is present. In my experience, intravenous urography is one of the most valuable recent assets which have come to our aid. In every case of renal calculus I make use of this excellent procedure. It shows whether there is dilatation in the opposite kidney and how the opaque medium is excreted by each kidney and thus gives valuable information about the renal functional activity. It likewise shows the parenchyma in many cases, and one can see if the good kidney is hypertrophied. If one carries out only one measure of investigation, let it be by X-rays with intravenous urography for preference.

As to operating on the kidney, the majority of single renal calculi are in the pelvis and therefore are removed by opening the pelvis posteriorly. Those situated in the calyces are reached by making an incision over the calyx which contains the stone; it is not necessary to open the whole kidney. The calyx is thinned out as a rule by back pressure, and for this reason usually there is little or no bleeding and such an operation is therefore not very hazardous. 
In certain cases it is necessary to take the kidney away. Nephrectomy is required when a kidney is so badly damaged that there is no likelihood that it will recover its normal configuration. When the calyces are considerably dilated and infection is superadded the patient will have ill-health for the rest of his life if such a kidney is left behind. In these circumstances if the other kidney is satisfactory one cannot do better than take the diseased one away. One is periodically faced with the problem: "Shall I take away the kidney or shall I merely remove the stone ?" If there is a doubt take the kidney away. If one's judgment is wrong in leaving the kidney, the patient is condemned to years of bad health, and one will probably have to do a nephrectomy later. I have performed a fair number of nephrectomies for renal calculus, and I have not regretted following this course. Success is largely a matter of having satisfied oneself about the other kidney. We have no knowledge that the expectation of life is reduced if a patient has only one sound kidney instead of two; this is a comforting fact. Amongst my own cases (40), I have not had any instance of stone occurring in the opposite kidney following nephrectomy. But some reliable authorities give the percentage of stone occurring in the remaining kidney as about 2 per cent. Even in bilateral cases in which only one kidney is grossly damaged, I have in a number of instances dealt with the less damaged organ by removing the stone, and then after giving the patient a rest for a month or more, I have removed the worse kidney. I have always noted great benefit from this as a result of the removal of extensive pyonephrosis. If the disease is equally distributed on both sides it follows that this method of treatment is contra-indicated.

\section{Ureteric Calculus.}

With regard to ureteric calculus, most of the stones in the ureter come from the kidney, and most of them get into the bladder. There is a certain part of the ureter where ninety per cent. of the stones held in the ureter find a resting place; that is opposite the ischial spine, just outside the bladder. It is about the most inaccessible part of the ureter as far as operation is concerned, but the majority of stones removed from the ureter by open operation are taken from this locality.

As to the clinical features of stone in the ureter, a certain number have colic in attacks which ultimately gives place to an aching in the loin from back pressure on the kidneys. There is a tendency for infection to be superadded with an exaggeration of the symptoms, and hæmaturia is fairly common with stone in the ureter. Every now and again one meets with a case of stone in the ureter which causes intense symptoms even though no stone is revealed by X-ray examination, as it may be too small to show on a radiogram. In these circumstances the stone is almost certain to pass into the bladder so that conservative treatment is indicated generally in the form of encouraging the patient to drink fluids copiously, so that a mechanical means of inducing the stone to move on is supplied. As to diagnosing shadows in the line of the ureter, a common pitfall is to mistake phleboliths in the true pelvic for ureteric calculi, and care must be taken in the discrimination. This can usually be done by means of an intravenous urogram, but the passage of an opaque catheter in conjunction with an X-ray examination may be required.

Expectant treatment is indicated in a number of cases, cystoscopic manipulations in others and ultimately in a few, there is need for open operation.

Before considering operation, if the stone is small enough to pass and expectant treatment has been undertaken for two weeks or more, one may assist 
the passage of the calculus by cystoscopic methods, such as slitting the ureteric orifice, passing catheters and injecting a local anæsthetic and lubricants. In properly chosen cases it is seldom that such methods fail.

In considering open operation on all my cases of stone in the upper urinary tract, I find that of a total of Ioo cases there was a mortality of 2 per cent. For all forms of surgical interference, that is to say, including open operations and cystoscopic manipulations in connection with ureteral calculus (I26 cases), my mortality was I.5 per cent., which shows that these cases can be dealt with very satisfactorily, even by operation. Fuller details are given in the following list:-

Mortality from surgical interference for calculous disease in the upper urinary tract.

Operations for unilateral Renal Calculus

No. of Cases. Deaths.

73

2

Pyelo and nephro-lithotomy

No. of Cases.

Deaths.

Pyelo and nephro-lithotomy (single kidney)

27

I

Nephrectomy

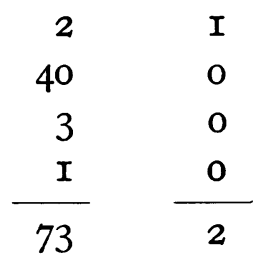

Nephro-ureterectomy
Hemi-nephrectomy
Operations for bilateral Calculus
Operations on both kidneys

13

Nephrectomy and lithotomy

Bilateral lithotomy

$\begin{array}{ll}2 & 0 \\ 4 & 0\end{array}$

Operations on one kidney only:

Lithotomy

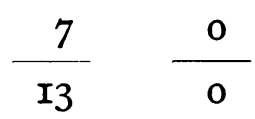

Open and cystoscopic operations for

Ureteric Calculus ... $\quad \ldots \quad \ldots$

Nephrectomy and nephro-

$\begin{array}{lllll}\text { Lithotomy } & \ldots & \ldots & \ldots & \ldots\end{array}$

Cystoscopic ureteral meatotomy or dilatation

$\cdots \quad \frac{26}{40} \quad \frac{0}{0}$

Percentage mortality for all open operations (IOO cases) $\quad \ldots \quad 2.0$

Percentage mortality for all forms of surgical interference $\quad \ldots \quad$ I.5 